\title{
Improved dosimetry in prostate brachytherapy using high resolution contrast enhanced magnetic resonance imaging: a feasibility study
}

\author{
Karen Buch, MD', Tye Morancy, MS², Irving Kaplan, MD², Muhammad M. Qureshi, MBBS³, Ariel E. Hirsch, MD³, \\ Neil M. Rofksy, MD4, Edward Holupka, PhD², Renee Oismueller, MD5, Robert Hawliczek, MD5, Thomas H. Helbich, MD', \\ B. Nicolas Bloch, MD \\ 'Department of Radiology, Boston University Medical Center, Boston, USA, ${ }^{2}$ Department of Radiation Oncology, Beth Israel Deaconess \\ Medical Center, Boston, USA, ${ }^{3}$ Department of Radiation Oncology, Boston University Medical Center, Boston, USA, ${ }^{4}$ Department of Radiology \\ University of Texas Southwestern, Dallas, USA, Institute for Radio-Oncology, Danube Hospital, Vienna, Austria, ${ }^{6}$ Department of Radiology, \\ Division of Gender and Molecular Imaging, Medical University Vienna, Vienna, Austria
}

\begin{abstract}
Purpose: To assess detailed dosimetry data for prostate and clinical relevant intra- and peri-prostatic structures including neurovascular bundles (NVB), urethra, and penile bulb (PB) from postbrachytherapy computed tomography (CT) versus high resolution contrast enhanced magnetic resonance imaging (HR-CEMRI).

Material and methods: Eleven postbrachytherapy prostate cancer patients underwent HR-CEMRI and CT imaging. Computed tomography and HR-CEMRI images were randomized and 2 independent expert readers created contours of prostate, intra- and peri-prostatic structures on each CT and HR-CEMRI scan for all 11 patients. Dosimetry data including $\mathrm{V}_{100}, \mathrm{D}_{90}$, and $\mathrm{D}_{100}$ was calculated from these contours.

Results: Mean $\mathrm{V}_{100}$ values from CT and HR-CEMRI contours were as follows: prostate $(98.5 \%$ and $96.2 \%, p=0.003)$, urethra $(81.0 \%$ and $88.7 \%, p=0.027)$, anterior rectal wall (ARW) $(8.9 \%$ and $2.8 \%, p<0.001)$, left NVB $(77.9 \%$ and $51.5 \%$, $p=0.002)$, right NVB $(69.2 \%$ and $43.1 \%, p=0.001)$, and PB $(0.09 \%$ and $11.4 \%, p=0.005)$. Mean $\mathrm{D}_{90}(\mathrm{~Gy})$ derived from CT and HR-CEMRI contours were: prostate (167.6 and 150.3, $p=0.012)$, urethra (81.6 and 109.4, $p=0.041)$, ARW (2.5 and $0.11, p=0.003)$, left NVB (98.2 and 58.6, $p=0.001)$, right NVB (87.5 and 55.5, $p=0.001)$, and PB (11.2 and 12.4, $p=0.554)$.

Conclusions: Findings of this study suggest that HR-CEMRI facilitates accurate and meaningful dosimetric assessment of prostate and clinically relevant structures, which is not possible with CT. Significant differences were seen between CT and HR-CEMRI, with volume overestimation of CT derived contours compared to HR-CEMRI.
\end{abstract}

Key words: brachytherapy, dosimetry, high resolution contrast enhanced MRI, prostate, prostate cancer.

\section{Purpose}

Transperineal prostate brachytherapy is one of the definitive treatment options for localized prostate cancer [1-4]. Post-implantation dosimetry is a crucial component of treatment as it enables assessment of the delivered dose to the prostate and peri-prostatic structures, assesses the position of the prostate implants, and gives feedback to the radiation oncologist regarding quality of the implantation technique $[1,3,5]$. In addition, potential areas of underdosing may be addressed with further implantation or external beam boost as appropriate [1].

Conventionally, post-brachytherapy data has been performed using computed tomography (CT) imaging largely because of its widespread availability, low cost, and convenience $[1,6]$. Soft tissue structures including the prostate gland, intra- and peri-prostatic structures are contoured on sequential transaxial images [1]. Dose-volume histograms (DVHs) are subsequently generated with visual representations of the isodose contours. Dosimetry is typically expressed as the parameter $\mathrm{D}_{90}$, defined as the minimum dose covering $90 \%$ of the prostate volume and is one of the most important predictive parameters for biochemical control in prostate cancer $[1,7]$.

There are many known challenges to defining the contours of prostatic and peri-prostatic structures on CT images given the relatively poor soft tissue contrast $[1,7,8]$. Increased inter-observer variability and variations in dosimetry results with increased doses delivered to the prostate were demonstrated with CT-derived contours [7-11]. While postbrachytherapy dosimetry is traditionally derived
Address for correspondence: Karen Buch, MD, Department of Radiology, Boston University Medical Center, Boston, USA, 820 Harrison Avenue, FGH bldg, 3rd Floor, Boston, MA 02118, USA,

phone: +1 617-414-2778, e-mail: Karen.buch@bmc.org
Received: 01.04 .2014

Accepted: 14.09.2014

Published: 30.12.2014 
from $\mathrm{CT}$, prior studies have described superior delineation of clinically relevant prostatic and peri-prostatic soft tissue structures on magnetic resonance imaging (MRI) compared to CT $[7,8]$. Specifically, T2-weighted MRI sequence offers superior soft tissue definition compared to CT and contours on T2-weighted MRI sequences have demonstrated greater reproducibility compared to CT-derived contours $[10,12]$. Additionally, prostate volume on T2-weighted MRI more closely approximates the volume assessed by transrectal ultrasound and volume at surgical resection [13-16].

We have developed a specialized high resolution, contrast enhanced MRI (HR-CEMRI) sequence optimized for the prostate gland, which is distinctly different from routine MRI examination. This HR-CEMRI sequence provides additional information to supplement the T2-weighted sequence with improved delineation of soft tissue structures, and a more accurate localization of brachytherapy seeds within the prostate and the identification of misplaced seeds within the peri-prostatic structures [8]. As previously reported, a specialized HR-CEMRI enables clear and robust delineation of brachytherapy seeds within a diffusely, contrast-enhanced, prostate gland while maintaining detailed borders of adjacent clinically relevant structures [8]. Treatment regimens derived from MRI-based dosimetry data may reduce complications to adjacent organs and structures including the rectum, penile bulb, and neurovascular bundles [13].

The purpose of this study was to evaluate prostatic and peri-prostatic dosimetry data from postbrachytherapy CT-based contours compared to HR-CEMRI-based contours. This study also builds off our prior work where we compared MRI-based contour using HR-CEMRI and T2-weighted sequences [8]. We hypothesized that HRCEMRI enables a more accurate dosimetry assessment based on its superior ability to discriminate the prostate, intra-, and peri-prostatic structures as compared to CT.

\section{Material and methods}

\section{Patient population}

Within a nine month period, 15 patients who were treated for prostate cancer with brachytherapy met the institutional inclusion criteria for prostate brachytherapy: 1) stage $\leq \mathrm{T} 2 ; 2$ ) positive findings in $<50 \%$ of biopsy cores; 3) prostate-specific antigen $<10 \mathrm{ng} / \mathrm{ml}$; 4) Gleason score < 7; and 5) prostate gland volume < 50 cc. All patients were treated with Palladium-103 implants (InterSource ${ }^{103}$, IBT InterSource, Mallinckrodt Medical B.V., a subsidiary of Covidien plc, Dublin, Ireland).

Exclusion criteria included: general contraindications for MRI (e.g. pacemaker, orbital shrapnel) and disorders interfering with endorectal coil examination (e.g. proctitis, extreme hemorrhoids, and active inflammatory bowel disease with rectal involvement).

Of the 15 patients, 11 patients were eligible to participate in this study under an IRB approved protocol, and standard informed consent was obtained. One of the patients refused to participate in the study and one patient was consented before MRI screening revealed that the patient had a pacemaker. Of the remaining 13 patients, two had HR-CEMRI data sets that were incompatible with the software platform used for dosimetry. The 11 enrolled patients received post-planning CT evaluations (standard of care) at three to five following seed placement. Post-treatment HR-CEMRI was also obtained at three to five weeks post-treatment. The CT and HR-CEMRI were performed within 10 days of one another (mean interval between CT and HR-CEMRI, 2 days; range: 9 days). All enrolled patients completed the study. These patients formed part of a cohort that has been previously reported [8].

\section{High resolution, contrast enhanced magnetic resonance imaging preparations}

Patients underwent rectal enema (Relaxyl Clyster, Nycomed-Amersham, a Unit of GE Healthcare, Chalfont St. Giles, UK) one to three hours before HR-CEMRI examinations. Local anesthesia of the anal region with topical lidocaine gel application was performed before the HR-CEMRI examinations (Xylocain 2\% Gel, AstraZeneca plc, London, UK). To reduce bowel peristalsis, $0.5 \mathrm{mg}$ Glucagon (GlucaGen ${ }^{\circledR}$, Novo Nordisk A/S, Bagsvaerd, Denmark) was administered i.v. just before the HR-CEMRI examinations and $0.5 \mathrm{mg}$ during the examinations (added to saline syringe in automated injection system).

\section{High resolution, contrast enhanced magnetic resonance imaging protocol}

All examinations were performed on a $1.5 \mathrm{~T}$ scanner with a pelvic phased-array surface coil (Magnetom Vision, Siemens AG, Munich, Germany) combined with a disposable endorectal prostate coil (eCoilT, MEDRAD, Inc., Pittsburgh PA, USA). The endorectal coil was connected to the pelvic phased-array surface coil, and combined images were obtained. All images were analytically corrected for the reception profile of the endorectal and pelvic phased-array coils [15]. Sagittal and transverse half-Fourier single-shot turbo spin-echo (TSE) sequences were first obtained to check coil position. High-resolution T1- and T2-weighted images were obtained in the transverse plane with a $16 \mathrm{~cm}$ field of view (FOV), matrix with 256 frequency-encoding steps, and 192 phase-encoding steps yielding in-plane spatial resolution of $0.63 \times 0.93 \mathrm{~mm}$, phase direction right-left, $100 \%$ phase over sampling. The T2-weighted sequence was a dual echo TSE sequence that was acquired from below the apex of the prostate to above the seminal vesicles with the following parameters: repetition time msec/first effective echo time msec/ second effective echo time msec $4000 / 83 / 165$, echo train length of 8.3, slice thickness (ST) $3 \mathrm{~mm}$, no intersection gap, 28 slices, three signals averaged (acquisition time: $10 \mathrm{~min}, 48 \mathrm{~s}$ ). T1-weighted images were acquired before and after contrast administration.

High resolution, contrast enhanced MRI imaging was performed after bolus injection of contrast media using a fast 3D-gradient echo sequence with a temporal resolution of $1 \mathrm{~min}, 35 \mathrm{~s}$. The imaging parameters included: repetition time msec/echo time msec of 8.1/4; flip angle of 18 degrees; FOV $160 \mathrm{~cm}$; matrix of $256 \times 192$; and ST $3 \mathrm{~mm}$, with no gap. Two precontrast and five postcon- 
trast acquisitions were obtained in succession with no delay between acquisitions. Gadopentetate dimeglumine (Magnevist ${ }^{\circledR}$, Bayer AG, Leverkusen, Germany) was injected as a bolus at a dose of $0.1 \mathrm{mmol} / \mathrm{kg}$ of body weight by an automated injection system (Spectris MRI Injection System, MEDRAD, Inc., Pittsburgh, PA, USA) at a rate of $4 \mathrm{ml} / \mathrm{s}$ during the last seconds of the second precontrast acquisition. This standardized time protocol assured that the injection of contrast was completed exactly before the first postcontrast acquisition started. The last time point data set was used to determine the number and locations of brachytherapy seeds. All examinations were supervised by one of two investigators, thereby ensuring consistency of the imaging protocol.

\section{Computed tomography protocol}

Non-contrast-enhanced CT was performed with a transverse section of $5 \mathrm{~mm}$ thickness with a pitch factor of 1.5 on a single-row spiral CT scanner (Somatom Emotion, Siemens AG, Munich, Germany), according to routine clinical protocol at our institution. Because we intended to compare the routine "standard of care" CT protocol with the HR-CEMRI, we did not match slice thickness; postimplantation dosimetric studies were routinely performed with $5 \mathrm{~mm}$ CT images.

\section{High resolution, contrast enhanced magnetic resonance imaging and computed tomography dosimetric analysis}

Transaxial CT and HR-CEMRI images were transferred to a computer workstation running the Interplant ${ }^{\circledR}$ $\mathrm{CT}$ based post-planning application (Elekta AB, Stockholm, Sweden). This algorithm is based on the Hough transform of the CT images to determine the location of the implanted radioactive seeds [17]. Two independent expert readers outlined the contours of the prostate gland, penile bulb (PB), anterior rectal wall (ARW), urethra, and bilateral neurovascular bundles (NVB) on each CT and HR-CEMRI for all 11 patients (total of 44 observations). Readers creating contours were a radiologist and a radiation oncologist trained by a radiologist for contouring. To prevent HR-CEMRI information from influencing the CT analyses, CT and HR-CEMRI images from all 11 patients were randomized so that the readers were blinded to the patient data. From these independent CT and HR-CEMRI contours, dosimetry was reported in terms of $\mathrm{D}_{90}, \mathrm{D}_{100}$ $\mathrm{V}_{100}, \mathrm{~V}_{120}$, and $\mathrm{V}_{150}$. $\mathrm{D}_{90}$ and $\mathrm{D}_{100}$ were defined as the minimum dose covering $90 \%$ and $100 \%$ of volume of prostate gland and clinically relevant intra- and peri-prostatic structures, respectively. $\mathrm{V}_{100}, \mathrm{~V}_{120}$, and $\mathrm{V}_{250}$ were defined as the percentage volume of the tissue receiving at least $100 \%$, $120 \%$, and $150 \%$ of the prescribed minimal peripheral dose $(\mathrm{mPD})$, respectively. These parameters were calculated for the prostate, urethra, $\mathrm{PB}, \mathrm{ARW}$, and bilateral NVB.

\section{Statistical analysis}

For each imaging modality (CT and HR-CEMRI), the data from the two readers was used to calculate a single mean value of a dosimetric parameter $\left(\mathrm{V}_{100}, \mathrm{~V}_{120}, \mathrm{~V}_{150}, \mathrm{D}_{90}\right.$, and $\mathrm{D}_{100}$ ) for every patient. Means and standard deviation are reported along with difference in CT and HR-CEMRI values (denoted as delta, $\Delta$ ). A linear mixed-effects model was chosen to account for within-patient correlation resulting from individual measurements from two different readers. A compound symmetry variance-covariance structure was used to model within-patient correlation. All statistical analyses were performed using SAS 9.1 software (SAS Institute, Cary, NC). A $P$ value less than 0.05 was determined statistically significant.

\section{Results}

\section{Prostate gland}

Statistically significant differences were noted in $\mathrm{V}_{100}$, $\mathrm{V}_{120}$, and $\mathrm{V}_{150}$ with a greater percentage of prostate volume receiving the prescribed dose in CT-derived contours compared to HR-CEMRI-derived contours, Table 1. Imaging examples are depicted in Figure 1. The mean ( \pm standard deviation) values from CT and HR-CEMRI contours, respectively, were as follows: $\mathrm{V}_{100}(98.5 \pm 1.5 \%$ and $96.2 \pm 3.6 \%, p=0.003), V_{120}(96.8 \pm 2.7 \%$ and 93.7 $\pm 4.8 \%, p=0.002)$, and $V_{150}(92.6 \pm 4.7 \%$ and $88.7 \pm 6.6 \%$, $p=0.002)$. Similarly, a higher minimum dose covering $90 \%$ and $100 \%$ of volume of prostate gland was noted in CT-derived prostate contours, Table 2: $\mathrm{D}_{90}(167.6 \pm 27.7$ and $150.3 \pm 35.5, p=0.012)$ and $\mathrm{D}_{100}(72.1 \pm 18.3$ and 53.9 $\pm 20.2, p=0.006$ ).

\section{Intra- and peri-prostatic structures}

A similar analysis was performed for intra- and periprostatic structures including urethra, ARW, right and left NVB, and PB. The mean $\mathrm{V}_{100}$ from CT and HR-CEMRI contours, respectively, was: urethra $(81.0 \pm 6.6 \%$ and $88.7 \pm 7.8 \%, p=0.027)$, ARW $(8.9 \pm 5.8 \%$ and $2.8 \pm 1.7 \%$, $p<0.001)$, left NVB $(77.9 \pm 21.9 \%$ and $51.5 \pm 18.0 \%$, $p=0.002)$, right NVB $(69.2 \pm 18.0 \%$ and $43.1 \pm 22.4 \%$, $p=0.001)$, and $\mathrm{PB}(0.09 \pm 0.3 \%$ and $11.4 \pm 17.2 \%, p=0.005)$. A similar statistically significant difference was noted in dosimetric parameters $\mathrm{V}_{120}$ and $\mathrm{V}_{150}$ for all peri-prostatic structures, Table 1.

The mean $\mathrm{D}_{90}$ derived from CT and HR-CEMRI contours, respectively, was: urethra $(81.6 \pm 19.3$ and 109.4 $\pm 31.5, p=0.041)$, ARW $(2.5 \pm 3.4$ and $0.11 \pm 0.05, p=0.003)$, left NVB $(98.2 \pm 33.9$ and $58.6 \pm 12.9, p=0.001)$, right NVB $(87.5 \pm 32.5$ and $55.5 \pm 20.3, p=0.001)$, and $\mathrm{PB}$ was $(11.2$ \pm 4.9 and $12.4 \pm 9.2, p=0.554)$. For the $\mathrm{PB}$, the analysis comparing minimum dose covering $100 \%$ of volume attained significance $(4.9 \pm 3.1$ and $2.6 \pm 2.6, p=0.03)$. Full results are presented in Table 2.

\section{Discussion}

Topographic knowledge and radiation dose delivered to the prostate and clinically relevant structures have implications in the appropriate management and treatment of prostate cancer. Accurate contouring for dosimetry data is vital to understanding potential dose-related complications to these structures. Damage to the penile bulb and adjacent erectile structures have been correlated with 
Table 1. Percentage volume of prostate gland and clinically relevant intra- and peri-prostatic structures receiving $100 \%, 120 \%$, and $150 \%$ of prescribed dose

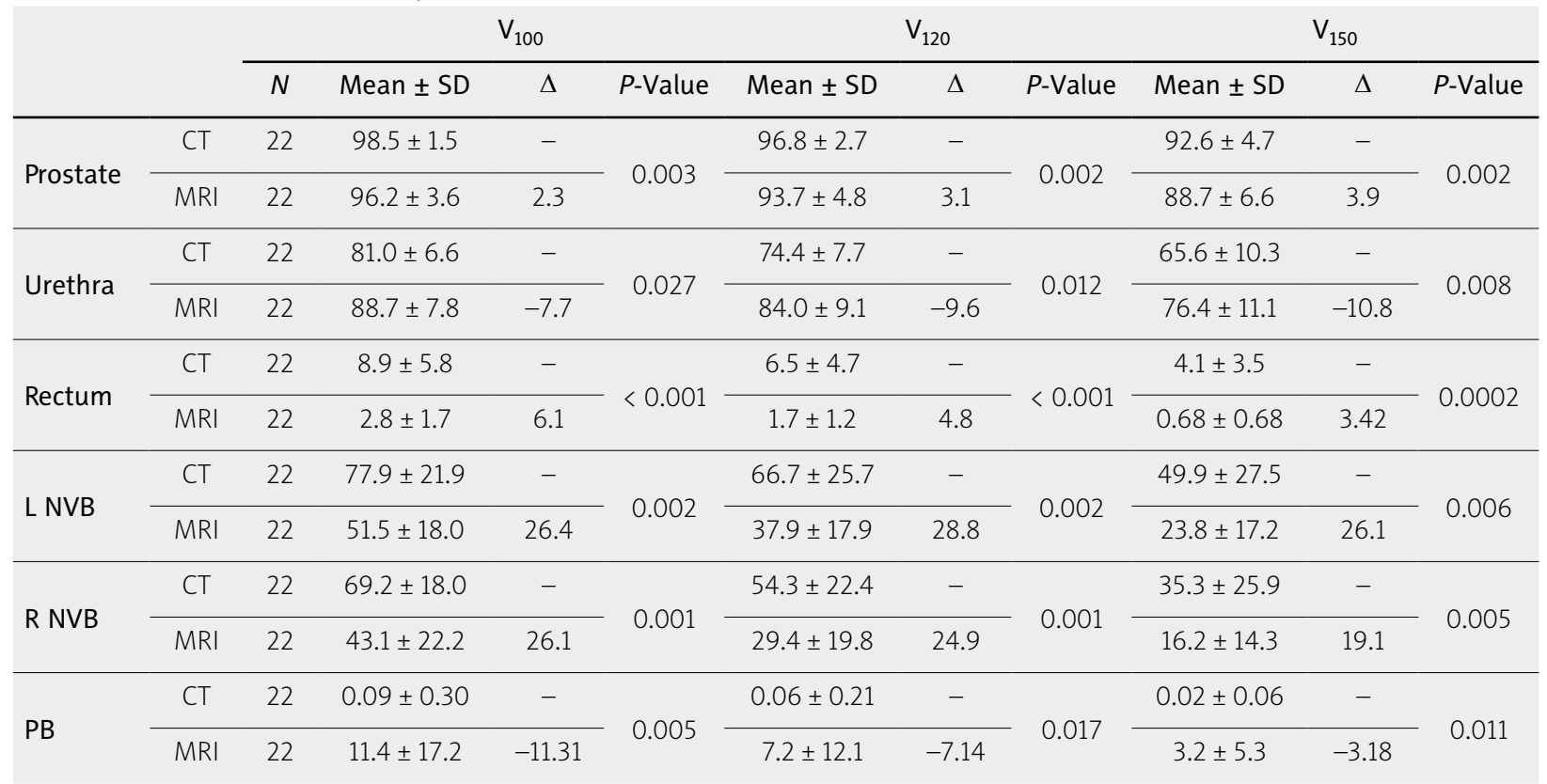

$N$-number of observations, SD-standard deviation, $\Delta$-difference between mean CT and MRI values, CT - computed tomography scan, MRI-magnetic resonance imaging, $L N V B$ - left neurovascular bundles, R NVB - right neurovascular bundles, $P B$ - penile bulb

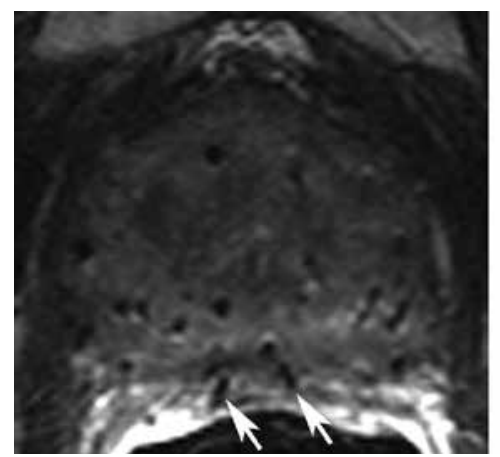

T2-weighted image

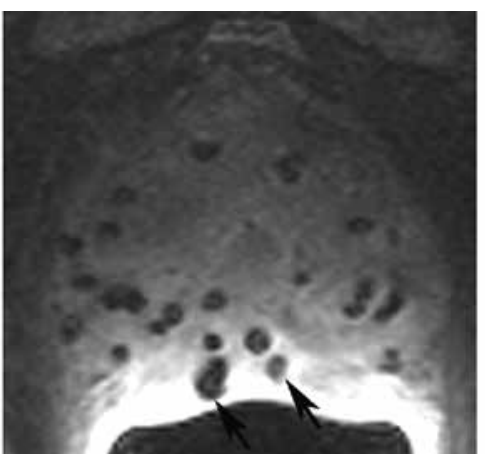

HR-CEMR image

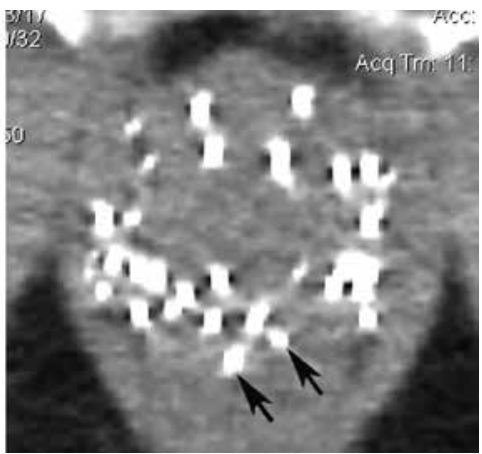

CT image

Fig. 1. Corresponding T2-W, HR-CEMR and CT images of the same location.

Please note the superior seed-tissue contrast of the HR-CEMR image: With the prostate gland homogeneously hyperintense (grey) and seeds causing signal voids (black), delineating two displaced seeds (arrows) in proximity to the Denonvillier's fascia/ rectal wall, which is not well appreciated on the CT image. T2-weighted image image does not visualize all of the seeds as clearly as the HR-CEMR image

erectile dysfunction, and damage to the neurovascular bundles has also been correlated with erectile dysfunction after radiation therapy [16].

The findings in this study are congruent and reflective of prior studies postulating that MRI based contours are superior to CT based contours for dosimetry assessments given the superior soft tissue contrast on MRI of these clinically relevant structures. This study expands upon prior studies utilizing HR-CEMRI based contours, which demonstrated strong, statistically significant differences in prostate dosimetry compared to CT. Greater $\mathrm{V}_{100}, \mathrm{~V}_{120}$, $\mathrm{V}_{150}, \mathrm{D}_{90}$, and $\mathrm{D}_{100}$ values were seen on $\mathrm{CT}$ derived contours compared to HR-CEMRI derived contours. A similar observation was seen for dosimetry of the rectum. Additionally, the level of statistical significance between
CT and HR-CEMRI-derived contours of the prostate and anterior rectal wall progressively increased over $\mathrm{V}_{100}$, $\mathrm{V}_{120}$, and $\mathrm{V}_{150}$ values. We believe that these results reflect an overestimation of the volumes of the prostate gland and anterior rectal wall based on CT due to its poor soft tissue contrast compared to HR-CEMRI. These differences are reflected in the larger $D_{90}$ and $D_{100}$ values for CT-derived contours of the prostate and anterior rectal wall compared to the HR-CEMRI derived contours of these structures.

The urethra and penile bulb were two structures where greater values of $V_{100}, V_{120}$, and $V_{150}$ were observed for HR-CEMRI derived contours. Smaller $\mathrm{V}_{100}, \mathrm{~V}_{120}$, and $\mathrm{V}_{150}$ values were observed in CT-derived contours of the urethra and penile bulb compared to HR-CEMRI-derived 
Table 2. Minimum dose (Gy) covering $90 \%$ and $100 \%$ of volume of prostate gland, and clinically relevant intraand peri-prostatic structures

\begin{tabular}{|c|c|c|c|c|c|c|c|c|}
\hline & & \multicolumn{4}{|c|}{$\mathrm{D}_{90}$} & \multicolumn{3}{|c|}{$D_{100}$} \\
\hline & & $N$ & Mean \pm SD & $\Delta$ & $P$-Value & Mean \pm SD & $\Delta$ & $P$-Value \\
\hline \multirow[t]{2}{*}{ Prostate } & CT & 22 & $167.6 \pm 27.7$ & - & \multirow{2}{*}{0.012} & $72.1 \pm 18.3$ & - & \multirow{2}{*}{0.006} \\
\hline & MRI & 22 & $150.3 \pm 35.5$ & 17.3 & & $53.9 \pm 20.2$ & 18.2 & \\
\hline \multirow[t]{2}{*}{ Urethra } & CT & 22 & $81.6 \pm 19.3$ & - & \multirow{2}{*}{0.041} & $48.6 \pm 15.7$ & - & \multirow{2}{*}{0.031} \\
\hline & MRI & 22 & $109.4 \pm 31.5$ & -27.8 & & $65.6 \pm 19.3$ & -17.0 & \\
\hline \multirow[t]{2}{*}{ Rectum } & CT & 22 & $2.5 \pm 3.4$ & - & \multirow{2}{*}{0.003} & $0.21 \pm 0.28$ & - & \multirow{2}{*}{0.099} \\
\hline & MRI & 22 & $0.11 \pm 0.05$ & 2.39 & & $0.10 \pm 0.0$ & 0.11 & \\
\hline \multirow[t]{2}{*}{ L NVB } & CT & 22 & $98.2 \pm 33.9$ & - & \multirow{2}{*}{0.001} & $64.7 \pm 24.6$ & - & \multirow{2}{*}{0.002} \\
\hline & MRI & 22 & $58.6 \pm 12.9$ & 39.6 & & $35.5 \pm 9.8$ & 29.2 & \\
\hline \multirow[t]{2}{*}{ R NVB } & CT & 22 & $87.5 \pm 32.5$ & - & \multirow{2}{*}{0.001} & $60.6 \pm 25.7$ & - & \multirow{2}{*}{0.001} \\
\hline & MRI & 22 & $55.5 \pm 20.3$ & 32.0 & & $34.5 \pm 15.1$ & 26.1 & \\
\hline \multirow[t]{2}{*}{ PB } & $\mathrm{CT}$ & 22 & $11.2 \pm 4.9$ & - & \multirow{2}{*}{0.554} & $4.9 \pm 3.1$ & - & \multirow{2}{*}{0.030} \\
\hline & MRI & 22 & $12.4 \pm 9.2$ & -1.2 & & $2.6 \pm 2.6$ & 2.3 & \\
\hline
\end{tabular}

contours. We postulate that these findings are due to limited soft tissue contrast on $\mathrm{CT}$ and a resulting inability to distinguish the borders of the prostatic urethra from the surrounding prostate gland and the penile bulb from the prostate apex.

Larger $V_{100}, V_{120}$, and $V_{150}$ values were observed for the neurovascular bundles on CT-derived contours compared to HR-CEMRI-derived contours; similarly, larger $D_{90}$ and $D_{100}$ values were also observed for CT-derived contours of the neurovascular bundles. We believe that while the location of the neurovascular bundles can be assessed on CT, the size and margins of the neurovascular bundles are not as clearly delineated when compared to HR-CEMRI-based contours.

The findings in our study are supported by prior studies whereby statistically significant differences between prostate volumes on CT compared to MRI were noted with prostate volumes on CT being 1.3 times larger than MRI [16,18-23]. MRI-delineated volume of the prostate is smaller compared to CT-delineated volume especially at the prostate apex attributed to improved differentiation of the prostate from the base of the seminal vesicles $[16,18]$. These studies also described an associated increased in CT-derived dosimetry compared to MRI-derived dosimetry in the prostate [18-22]. Debois et al. investigated differences in dosimetry data derived from CT and MRI noting that the volume of the rectum receiving $80 \%$ of the prescribed dose was smaller for the treatment plans using MRI-based assessment compared to a CT-based assessment [20]. Sannazzari et al. evaluated differences in dosimetry derived from CT versus MRI noting that approximately $10 \%$ of the rectal volume could be spared when using MRI for prostate delineation [21]. Additionally, Krempien et al. showed that the mean dose delivered to the rectum could be reduced from $74.9 \%$ to $64.2 \%$ of the prescribed dose using MRI-based contours compared to CT-based contours [22]. This study also builds off of our prior research which demonstrated the added value of using HR-CEMRI, and T2-weight sequences for improved targeted seed implantation analysis and enhanced quality control [8]. The advantage of using a HR-CEMRI protocol is the late contrast phase of enhancement, which provides improved localization of seeds and highly detailed delineations of the prostate gland and adjacent clinically relevant intra- and peri-prostatic structures.

In a recently published study, Ohashi et al. compared differences in dosimetry data for the prostate gland from CT- and CE-MRI-derived contours [24]. In that study, there were no statistically significant differences between the $D_{90}$ and $V_{100}$ estimated by CT-based and MRI-based dosimetry. Using manual seed detection, Ohashi et al. evaluated CT and MRI-based dosimetry for the prostate gland only, clinically relevant intra and peri-prostatic structures were not included in the dosimetric assessment [24]. In contradistinction, we used an automated seed detector for calculating CT- and MRI-based dosimetry minimizing counting errors and potential reader bias, a process which we believe is likely to provide more accurate dosimetry data. We have also expanded upon the study of Ohashi et al. by evaluating dosimetry of the adjacent clinically relevant intra- and peri-prostatic structures. Dosimetry of these clinically relevant structures is imperative for minimizing potential radiation-induced morbidity and identifying patients potentially at risk for post-brachytherapy complications. Detailed information regarding brachytherapy seed locations and knowledge of radiation dose delivered to adjacent organs can be addressed earlier through medical or interventional treatment, and the patient can be prepared for certain side 
effects. For example, several seeds placed close to the rectal wall, the patient can be prepared for diarrhea and patients can be followed more closely to avoid complications from rectal bleeding and diarrhea.

Conventionally, post-treatment dosimetry has been based on CT-derived contours, however, a prior study described an overestimation of prostate volume attributed to impaired delineation of the prostate from the anterior rectal wall which if used for pre-treatment planning purposes could potentially result in an increased radiation dose to the rectum and increased potential for post-radiation toxicity [8]. Therefore, dose limitation to these clinically and functionally relevant intra- and peri-prostatic structures is important for reducing overall patient morbidity $[7,8]$. Despite these known limitations, CT remains the modality of choice for post-brachytherapy dosimetry in most practices [8,9]. Prior studies have demonstrated numerous advantages to using prostate MRI for pre-treatment planning including reduced toxicity and may alter the side affect profile $[25,26]$. Performing pre-treatment and early post-treatment, prostate MRI could ultimately lead to lower patient morbidity and improved clinical outcomes.

This study has some recognized limitations. Firstly, our sample size is relatively small and a larger scale study should be performed before extrapolation to a generalized population. Secondly, we could not prove the actual volumes of the prostate and adjacent structures (urethra, ARW, NVB, and PB) since we did not have a reference standard, as our patients did not undergo surgery and excised gross pathology specimens were therefore unavailable. Instead, we relied upon the results of two independent, blinded readers in a retrospective consensus to provide a reference standard for this study. Thirdly, HR-CEMRI was performed using a $3 \mathrm{~mm}$ slice thickness and CT was performed using $5 \mathrm{~mm}$ slice thickness. We believe that the impact of using slightly different slice thicknesses is small as the number of seeds detected on CT and MRI were similar. Therefore, we believe that the difference in slice thickness between HR-CEMRI and CT was sufficient to detect the seeds and to perform the dosimetry studies. For some structures such as the prostate gland, CT overestimates the volume of the prostate gland and underestimates the volume of adjacent clinically relevant peri-prostatic structures. We believe that using a CT slice thickness of $3 \mathrm{~mm}$ would not improve the shortcomings of $\mathrm{CT}$, and that difference in calculated volumes and dosimetry data is secondary to the limited soft tissue contrast and restricted ability to delineate the prostate capsule and adjacent structures. Lastly, there may be slight spatial distortion by the endorectal coil on HR-CEMRI. On the other hand, this distortion is predictable and may be taken into account for volume measurements. The use of 3-Tesla HR-CEMRI and an external phased array coil is attractive for patient convenience and would eliminate this potential area of distortion, however, further investigation is needed.

In conclusion, these results demonstrate that there are differences in CT- and HR-CEMRI-derived volumes and calculated doses delivered to the prostate gland and adjacent clinically relevant structures. Statistically significant differences between adjacent intra- and peri-prostatic structures on CT compared to HR-CEMRI-based contours are felt to result from the superior soft tissue contrast on HR-CEMRI, particularly using a sequence obtained in the late enhancement phase over conventional CT. Accurate dosimetric assessment of these clinically and functionally relevant structures is critical to guiding future treatments and reducing overall patient morbidity. The results of this study suggest that HR-CEMRI is superior to CT for the calculation of post-brachytherapy dosimetry data for the prostate, and the intra- and peri-prostatic structures. Post-brachytherapy dosimetry can serve as a quality control to the implant done which may translate into the clinical outcomes. While this study was specifically investigating brachytherapy dosimetry, we believe that our findings will be useful to guide treatment choices for prostate cancer patients.

\section{Disclosure}

Authors report no conflict of interest.

\section{References}

1. Acher P, Rhode K, Morris S et al. Comparison of combined $\mathrm{x}$-ray radiography and magnetic resonance (XMR) imaging-versus computed tomography-based dosimetry for the evaluation of permanent prostate brachytherapy implants. Int J Radiat Oncol Biol Phys 2008; 71: 1518-1525.

2. Aronowitz JN, Rivard MJ. The phylogeny of permanent prostate brachytherapy. J Contemp Brachytherapy 2013; 5: 89-92.

3. Skowronek J. Low-dose-rate or high-dose-rate brachytherapy in treatment of prostate cancer - between options. J Contemp Brachytherapy 2013; 5: 33-41.

4. Salembier C, Rijnders A, Henry A et al. Prospective multicenter dosimetry study of low-dose Iodine- 125 prostate brachytherapy performed after transurethral resection. J Contemp Brachytherapy 2013; 5: 63-69.

5. Ash D, Flynn A, Battermann J et al. ESTRO/EAU/EORTC recommendations on permanent seed implantation for localized prostate cancer. Radiother Oncol 2000; 57: 315-321.

6. Ohashi T, Momma T, Yamashita $S$ et al. Dosimetric effects of prone and supine positions on post-implant assessments for prostate brachytherapy. J Contemp Brachytherapy 2013; 5: 122-126.

7. Maletz KL, Ennis RD, Ostenson J et al. Comparison of CT and MR-CT fusion for prostate post-implant dosimetry. Int J Radiat Oncol Biol Phys 2012; 82: 1912-1917.

8. Bloch BN, Lenkinski RE, Helbich TH et al. Prostate postbrachytherapy seed distribution: comparison of high-resolution, contrast-enhanced, T1- and T2-weighted endorectal magnetic resonance imaging versus computed tomography: initial experience. Int J Radiat Oncol Biol Phys 2007; 69: 70-78.

9. Khoo VS, Adams EJ, Saran F et al. A Comparison of clinical target volumes determined by CT and MRI for the radiotherapy planning of base of skull meningiomas. Int J Radiat Oncol Biol Phys 2000; 46: 1309-1317.

10. Chen L, Price RA, Jr., Wang L et al. MRI-based treatment planning for radiotherapy: dosimetric verification for prostate IMRT. Int J Radiat Oncol Biol Phys 2004; 60: 636-647.

11. Croke J, Malone S, Roustan Delatour N et al. Postoperative radiotherapy in prostate cancer: the case of the missing target. Int J Radiat Oncol Biol Phys 2012; 83: 1160-1168.

12. McLaughlin PW, Evans C, Feng M et al. Radiographic and anatomic basis for prostate contouring errors and methods to 
improve prostate contouring accuracy. Int J Radiat Oncol Biol Phys 2010; 76: 369-378.

13. Polo A, Cattani F, Vavassori A et al. MR and CT image fusion for postimplant analysis in permanent prostate seed implants. Int J Radiat Oncol Biol Phys 2004; 60: 1572-1579.

14. Tanaka O, Hayashi S, Matsuo M et al. Comparison of MRIbased and CT/MRI fusion-based postimplant dosimetric analysis of prostate brachytherapy. Int J Radiat Oncol Biol Phys 2006; 66: 597-602.

15. Scheidler J, Hricak H, Vigneron DB et al. Prostate cancer: localization with three-dimensional proton MR spectroscopic imaging - clinicopathologic study. Radiology 1999; 213: 473480.

16. Steenbakkers RJ, Deurloo KE, Nowak PJ et al. Reduction of dose delivered to the rectum and bulb of the penis using MRI delineation for radiotherapy of the prostate. Int J Radiat Oncol Biol Phys 2003; 57: 1269-1279.

17. Holupka EJ, Meskell PM, Burdette EC et al. An automatic seed finder for brachytherapy CT postplans based on the Hough transform. Med Phys 2004; 31: 2672-2679.

18. Rasch C, Barillot I, Remeijer P et al. Definition of the prostate in CT and MRI: a multi-observer study. Int J Radiat Oncol Biol Phys 1999; 43: 57-66.

19. Roach M, 3rd, Faillace-Akazawa $P$, Malfatti $C$ et al. Prostate volumes defined by magnetic resonance imaging and computerized tomographic scans for three-dimensional conformal radiotherapy. Int J Radiat Oncol Biol Phys 1996; 35: 10111018.

20. Debois M, Oyen R, Maes F et al. The contribution of magnetic resonance imaging to the three-dimensional treatment planning of localized prostate cancer. Int J Radiat Oncol Biol Phys 1999; 45: 857-865.

21. Sannazzari GL, Ragona R, Ruo Redda MG et al. CT-MRI image fusion for delineation of volumes in three-dimensional conformal radiation therapy in the treatment of localized prostate cancer. Br J Radiol 2002; 75: 603-607.

22. Krempien RC, Schubert K, Zierhut D et al. Open low-field magnetic resonance imaging in radiation therapy treatment planning. Int J Radiat Oncol Biol Phys 2002; 53: 1350-1360.

23. McLaughlin PW, Troyer S, Berri S et al. Functional anatomy of the prostate: implications for treatment planning. Int J Radiat Oncol Biol Phys 2005; 63: 479-491.

24. Ohashi T, Momma T, Yamashita S et al. Impact of MRI-based postimplant dosimetric assessment in prostate brachytherapy using contrast-enhanced T1-weighted images. Brachytherapy 2012; 11: 468-475.

25. Dinkla AM, Pieters BR, Koedooder K et al. Improved tumour control probability with MRI-based prostate brachytherapy treatment planning. Acta Oncol 2013; 52: 658-665.

26. Albert JM, Swanson DA, Pugh TJ et al. Magnetic resonance imaging-based treatment planning for prostate brachytherapy. Brachytherapy 2013; 12: 30-37. 